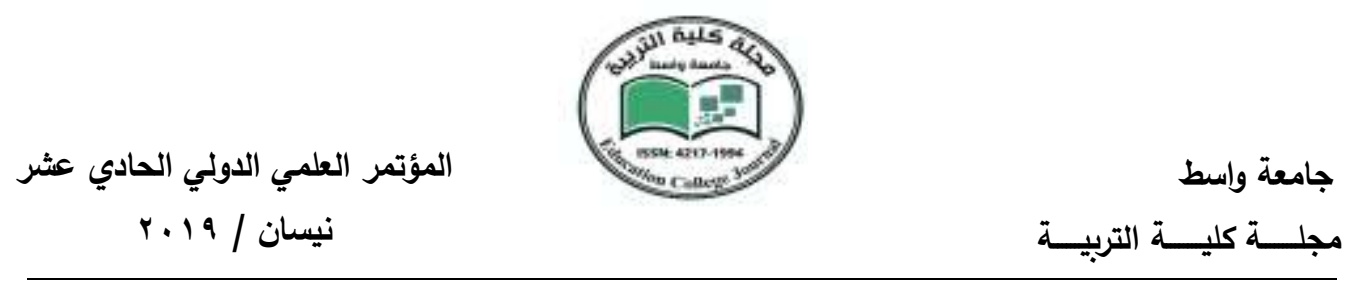

الثرق الاوسط الجديد في منظور الاستراتيجية الامريكية

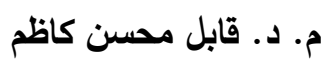

المديرية العامة لتربية ذي قار / تربية الرفاعي

الملخص:

شكل الإحتلال الأمريكي للعراق خروجا على لعلاقات الدولية التي عرفها العالم في أعقاب الحرب

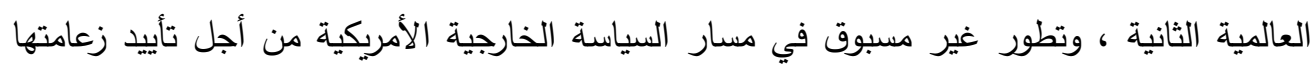
الأحادية على العالم ، وقد مثل لشرق الأوسط نقطة الإرتكاز في الإستهداف الإستراتيجي الأمريكي .

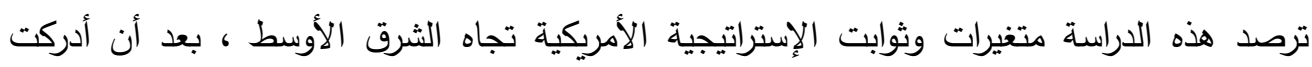

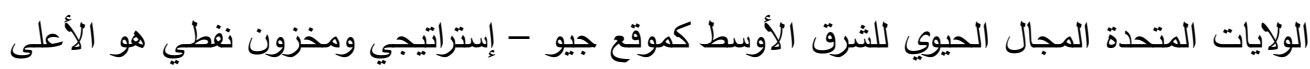

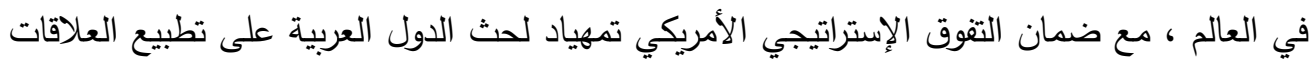

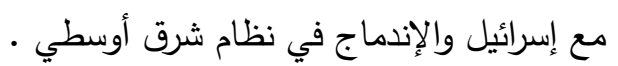
تعمل الدراسة على إثارة الدلالات العلمية التي يمكن ان تكون بمثابة الحوافز المطلوبة لخلق إستجابة

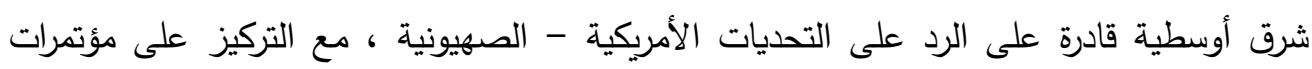

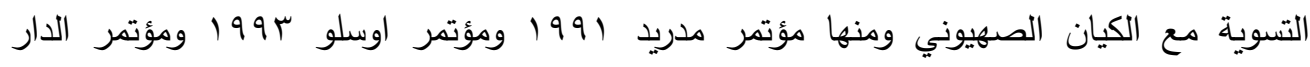

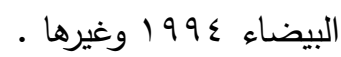



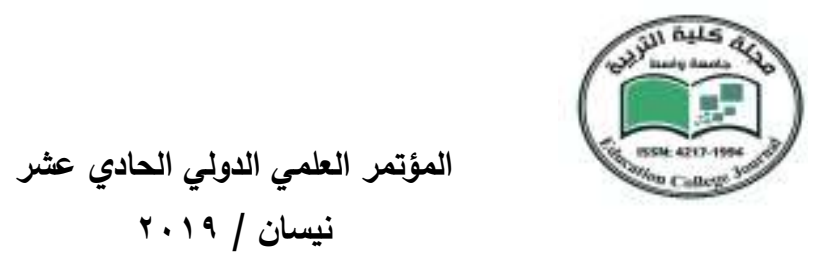

جامعة واسط

مجلــــة كليــــة التربيــــة

Summary

The US occupation of Iraq was a departure from the international relations that the world witnessed in the wake of the Second World War and an unprecedented development in the course of US foreign policy in order to support its unilateral leadership over the world, and represented the Middle East as a focal point for American strategic targeting.

This study examines the variables and constants of the US strategy towards the Middle East, after the United States realized the vital field of the Middle East as a geo-strategic location and the highest oil reserves in the world, while ensuring strategic American superiority. .

The study aims to raise the scientific connotations that could serve as the incentives needed to create a Middle Eastern response capable of responding to the American-Zionist challenges, with a focus on settlement conferences with the Zionist entity such as the 1991 Madrid Conference, the 1993 Oslo Conference and the 1994 Casablanca Conference.

المقدمة

اظهرت الحرب العالمية الثانية الاهمية الاستراتيجية لمنطقة الثرق الاوسط، وتعزز ذلك عندما صار واضحا بان اعادة بناء اوربا واليابان وتحقيق الانتعاش الاقتصادي في الولايات المتحدة الامريكية في اعتاب الحرب سيعتمد على ثروات الثرق الاوسط والمتمثلة بالنفط، والذي يمثل ثلثي مخزون النفط في العالم، مما لا شك فيه صارت الاهمية السياسية للمنطقة تكبر في اعين واضعي السياسة الامريكية وباتت اهمية الثرق الاوسط مرتبطة في الوقت ذاته بالحرب الباردة بين الولايات المتحدة والاتحاد السوفييتي، لذا صار ضروريا على الولايات المتحدة الامريكية استعمال كل الاساليب لاجل منع الاتحاد السوفييتي من مد نفوذه في المنطقة بعد ضعف كل من بريطانيا وفرنسا، كما ساعد ظهور اسرائيل عام 919 19 على زيادة الاهتمام الامريكي في الثرق الاوسط.

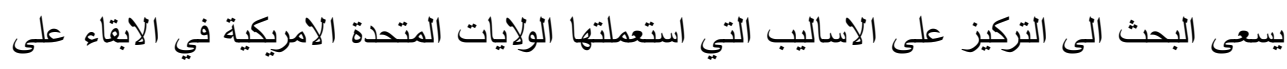

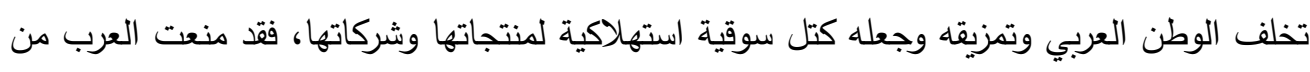


المؤتمر العلمي الاولي الحادي عثر

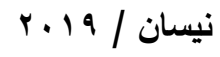

جامعة واسط

مجلــــة كليـــة التربيـــة

امتلاك التكنولوجيا، ومنعت قيام اي محطة نووية للاغراض السلمية، كما انها خرجت من القانون الدولي الذي يمنع اسقاط اي نظام سياسي بالقوة العكرية والاحتلال العسكري المباشر ، فكان احتلال العراق لا يستند الى اي مبرر او مسوغ قانوني، فضلا عن خروجها على منظمة الامم المتحدة التي لتهي لم تمنح الولايات المتحدة اي تفويض بشن الحرب على العراق واحتلاله، وهنا تكمن المشكلة والخطورة، اذ يجعل ذلك ان تواجه هذه المنظمة المصير نفسه الذي واجهته عصبة الامم بعد الحرب لهب العالمية الاولى. يركز البحث على سياسة النفوذ الامريكي بتزعم العالم وقيام الامبراطورية الامبريالية، التي بدأت

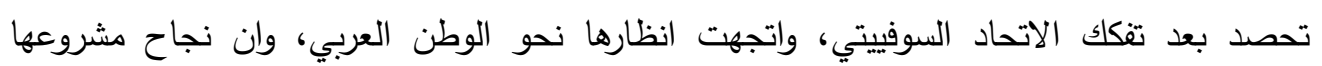

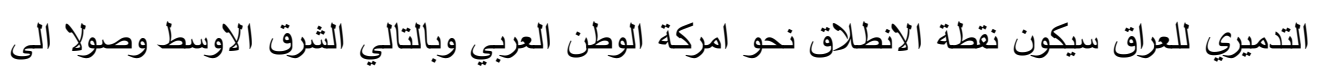
امركة العالم عبر مخطط العولمة الامريكي.

\section{الثرق الاوسط الجديد في منظور الاستراتيجية الامريكية}

الشرق الاوسط: مصطلح جغرافي يشمل المنطقة الممتدة لمساحة جغرافية تقدر بحوالي IV,VVA مليون كم ب وتشمل الوطن العربي وباكستان وافغانستان وايران وتركيا وقبرص وجزر القدر (')، وتثكل

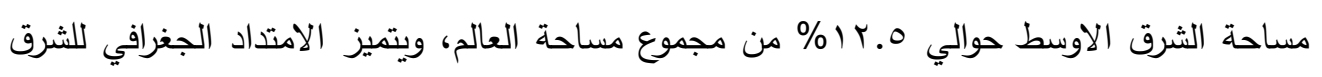

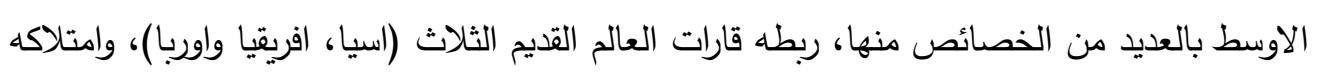

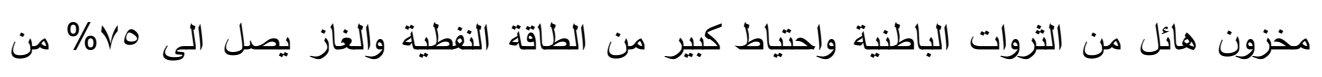
اجمالي طاقة العالم، ويمتلك الشرق الاوسط من المنافذ البحرية تمتد من بحر قزوين الى البحر الاسود الى الخليج العربي فالمحيط الاطلسي والى البحر المتوسط والاحمر (؟).

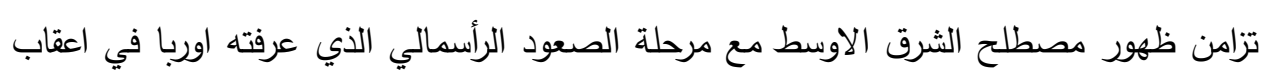
الثورة الصناعية، اذ ان الدول الرأسمالية الاوربية اخذت تفتش عن مناطق جديدة للحصول على المواد

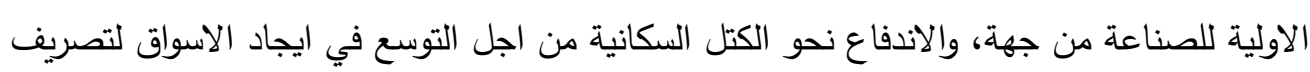
فائضها السلعي من جهة ثانية(") الأنة 
المؤتمر العلمي الاولي الحادي عشر

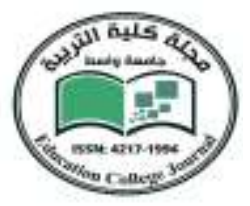

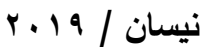

جامعة واسط

مجلــــة كليــــة التربيــــة

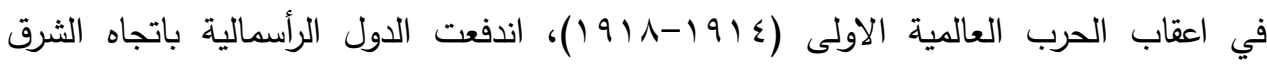
الاوسط، واستمرت حتى اواخر القرن العشرين لتدخل حالياً عصر العولمة(๕)، وقد عدت الدول

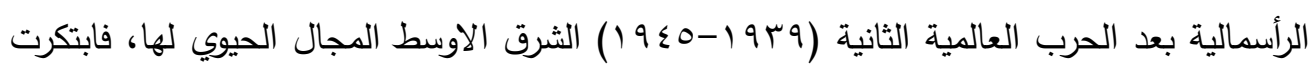
وسائل عديدة للسيطرة على المنطقة والحاقها بهم، والعمل على تخلفها، وفي عام 9 أح كان الانجاز

المتقدم للغرب هو تأسيسه الكيان الصهيوني لاجل الامساك بالمنطقة والسيطرة على ثرواتها(ْ). لم يكن مشروع الشرق الاوسط فكرة طارئة في السياسة الخارجية الامريكية، بل ثابت استراتيجي في كل الحكومات التي تعاقبت على رئاسة الولايات المتحدة منذ نهاية الحرب العالمية الثانية، اذ ان الولايات المتحدة كانت تهدف الى شرق اوسط يكون بيدها بديلا للنظام الاقليمي العربي بعد ولادة جامعة الدول العربية 0؛9 1، كما انها تسعى الى جعل الكيان الصهيوني ليس دخيلا على المنطقة واتجهت لتسويقه في فضاء عربي اسلامي، فضلا عن جعل اسرائيل ذات وزن تكنولوجي واقتصادي،

وبذلك فان هذا المشروع هو صهيو -امريكي(؟).

تمثلت الخطوة الرئيسية نحو شرق اوسطي جديد باخراج مصر من المنظومة العربية بدءا بزيارة السادات للقدس 9VV و وتوقيعه اتفاقية كامب-ديفيد 9VA 19 مما افقد مصر وزنها القومي، وافقد النظام العربي توازنه الاقليمي والدولي(')، وفي اواسط الثمانينيات ظهرت للعلن الخطوة الاولى لمشروع شرق اوسط جديد، اذ أعد شمعون بيريز (مشروع مارشال للشرق الاوسط)، واطلق عليه البعض (مشروع خليل-بيريز) بعد اشتراك مصطفى خليل رئيس الحزب الوطني في مصر في المشروع، ونص المشروع على استيعاب الثروات الخليجية ودمجها مع اقتصاديات الغرب ضمن برنامج تديره الولايات المتحدة الامريكية(^).

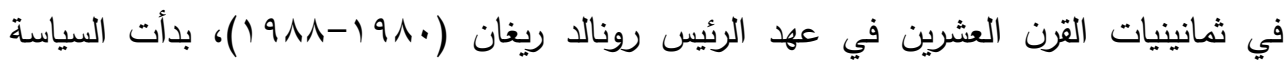
الخارجية الامريكية تخرج على سياسة الاحتواء للاتحاد السوفييتي والقوى الممانعة الاخرى للسياسة الامريكية، واتجهت الى سياسة القضاء على الاتحاد السوفييتي وسائر القوى الاخرى، كما جرى زيادة في الميزانية العسكرية الامريكية بنسبة جا \%٪، فضلا عن الاسراع في نظم نووية جديدة، مما يعني مركزية الخيار العسكري في تحقيق اهداف السياسة الامريكية(9). 
المؤتمر العلمي الاولي الحادي عشر

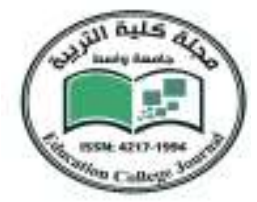

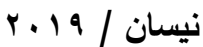

\section{جامعة واسط}

مجلـــة كليـــة التربيــة

سعت الولايات المتحدة الى بلورة مشروع امريكي صهيوني في عهر الرئيس ريغان يقضي بتفكيك عدة دول عربية في منطقة الثرق الاوسط بهدف تحقيق ما يسمى باسرائيل الكبرى من النيل الى الفرات، وتحقيق الهيمنة السياسية والاقتصادية الامريكية على ثروات المنطقة العربية، ثم التمهيد لقيام نظام شرق اوسطي يحل محل النظام العربي الراهن وينهي الرابطة العربية، ويفضي ذلك بتقسيم منطقة الثرق الاوسط مما فيها تركيا وايران وافغانستان، ورسم خارطة جديدة للمنطقة يكون فيها لكل مذهب ديني وعرق عنصري اقليم خاص به، ولكل قبيلة في الجزيرة العببية دويلة(· '). استغلت الولايات المتحدة الامريكية سقوط الاتحاد السوفييتي عام •991، فسارعت الى الامساك بعصب القوى الاقتصادية المتمثلة بثروات الخليج العربي، فضلا عن عمل الادارة الامريكية الى اعادة صياغة عملية تدويل أمن الخليج العربي بعيدا عن نظام القطبية الثنائية، والاتجاه نحو القطبية

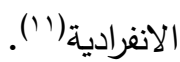
نجحت الولايات المتحدة الامريكية في تحشيد جيوش اكثر من ثلاثين دولة فيها عدد من الدول العربية ابان الاحتلال العراقي للكويت، واجبار العراق للامتثال لقرار (†^^)(ז')، وبالتالي اخراج العراق من منظومة القوة العسكرية والاقتصادية، وساعد ذلك الولايات المتحدة على الامساك بالنفط

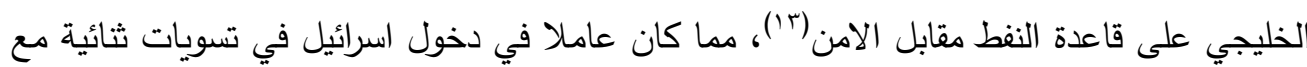
الدول العربية، وهذا ما تمخض عن مفاوضات مؤتمر مدريد 1991، الذي اكد الى المضي في عملية التسوية، مما عكس تحولا عربيا نحو الاندماج في نظام شرق اوسطي، وارتبط ذلك بالمخططات الامريكية-الاسرائيلية التي تهدف الى القضاء على النظام العربي وتهميش دور الجامعة العربية بوصفها مؤسسة للنظام الاقليمي العربي في مفاوضات التسوية مع اسرائيل(\&ٔ). ان مشروع الثرق الاوسط الجديد في منظور الولايات المتحدة الامريكية والكيان الاسرائيلي يظهر خطره ليس لكونه يقوم على انقاض النظام العربي، وانما يجعل اسرائيل ذات مركز سياسي واقتصادي واستراتيجي، وظهر ذلك في العديد من المؤتمرات، ففي مؤتمر مدريد ا991، تم التمهيد لاستيعاب المنطقة العربية ضمن نظام الثرق الاوسط الجديد، بحيث يتمكن الغرب من السيطرة على موارد

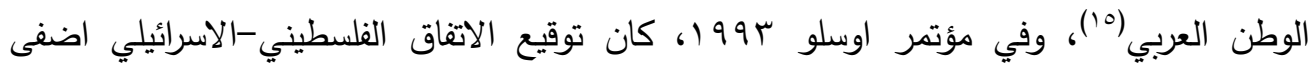


المؤتمر العلمي الاولي الحادي عشر

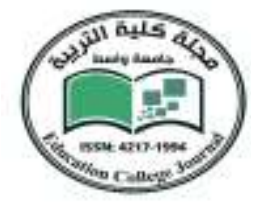

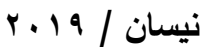

\section{جامعة واسط} مجلـــة كليـــة التربيـــة

الصفة الثرعية على الوجود الاسرائيلي، كما انه حدثا غير مسبوق، اذ يعد تتازل من اصحاب الارض الحقيقيين وهم الفلسطينيين قبل ان يكون حقا قوميا عربيا، كما انه كسر حاجز التردد عند بعض الدول العربية للانضمام للتكتلات الثرق اوسطية الجديدة(1'). ابرمت الاردن عام ع99 اتفاقا مع اسرائيل في وادي عربة، وكان الاتفاق بصيغة معاهدة، مما اضفى تطبيعا خاصا مع اسرائيل، لاسيما وان الاردن مركب سكاني اردني وفلسطيني، ترتب على هذا الاتفاقيات (مدريد واوسلو ومعاهدة الاردن) ضغط امريكي على دول الخليج العربي لالغاء مقاطعتها لاسرائيل، وقد التقى وزير الخارجية الامريكي (وارن كريستوفر) مع وزراء خارجية مجلس التعاون لدول الخليج العربي لهذا الغرض، وقد وافقوا على رفع المقاطعة غير المباشرة مع اسرائيل واصدروا بيانا بهذا الثأن(V)، وبذلك نجحت الولايات الدتحدة الامريكية في تذليل عقبات الاعتراض على مشروع

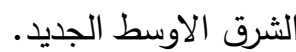
في تثرين الثاني ؟99 اعد مؤتمر في مدينة الدار البيضاء المغربية، وكان المؤتمر في ظاهره اقتصادي، لكن كان مجرد غطاء لانجاز تفاهمات سياسية بين الاطراف المشاركة والتي بلغت (آ) دولة، لاسيما بين العرب والاسرائليين، وقد رأس المؤتمر الملك (الحسن الثاني) وكان برعاية الرئيس الامريكي (بيل كلنتون) والرئيس الروسي (يلتسين)(^)، وكانت فكرة شرق اوسط جديد هي السائدة بين الرئيسين الامريكي والروسي، ووقع الوفد الامريكي الى اقامة مشاريع مشتركة بين اسرائيل والاقطار العربية، فضلا عن رفع المقاطعة الاقتصادية العربية لاسرائيل، وكان موقف الولايات المتحدة تجاه العلاقات العربية الاسرائيلية يرتكز على مفهومين للسلام تمثلا في السلام السياسي والسلام الاقتصادي(19) سعت الولايات المتحدة الامريكية السيطرة المباشرة على المنطقة، لاسيما الخليج العربي، والغاء النظام القديم القائم على العلاقات التقليدية بالنظم العببية الحليفة، وعدت ذلك الركيزة الاساسية لقيام الثرق الاوسط الجديد، لذا بدأت الولايات المتحدة الامريكية القيام بالحرب الاستباقية وتمكنت من احتلال افغانستان عام /...r والعراق عام r...r، وقد مثل احتلال افغانستان حربا دفاعية على ساحة العدو (القاعدة)، اما احتلال العراق فكان نموذجا هجوميا من دون اي مرتكزات تبريرية كان القصد منه نظام اقليمي جديد او السيطرة المباشرة من جانب النظام الدولي القائم على 
المؤتمر العلمي الاولي الحادي عشر

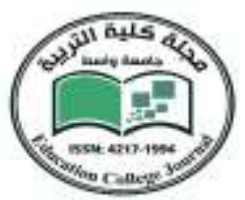

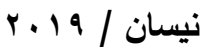

جامعة واسط

مجلـــة كليــة التربيـة فيسة

الهيمنة الانفرادية، كما انه امتداد للاهداف والمحددات الاساسية للسياسة الامريكية في الثرق الاوسط

بصورة عامة والخليج العربي بصورة خاصة(·r).

سعت الولايات المتحدة من خلال عملية السيطرة على المنطقة في هذه المرحلة الى تحقيق العديد

من الاهداف لعل ابرزها حماية امن اسرائيل وجعلها تتفوق على الدول العربية في كل المجالات، فضلا عن السيطرة الامريكية المباشرة على منابع النفط وموانئ التصدير والتحكم في اسعاره وتسويقه(r)، وترتب على هذه السياسة انه صار لاسرائيل الدور المباشر في ادارة سياسة المنطقة. من الركائز الاساسية لقيام نظام شرق اوسطي جديد يستجيب للتوجهات الامريكية هو الانقلاب على العلاقات الامريكية التقليدية بالنظم العربية الحليفة والسعي لتغييرها بما يتلائم مع التطورات في المنطقة والمصالح الامريكية الاسرائيلية(r). في عام \&.. ب عقد مؤتمر مجموعة الدول الصناعية الثماني، وفيه تقدمت الولايات المتحدة الامريكية بوثيقة عنوانها (مشروع الثرق الاوسط الجديد)، اكدت فيه ان المنطقة العربية تعاني من غياب الحرية وبطء المعرفة وتدني الانتاجية، اذ ان اجمالي الناتج القومي العربي لدول الجامعة العربية مجتمعة هو اقل من ناتج دولة اوربية واحدة وهي اسبانيا ونصف ناتج ايطاليا، كما تعاني المنطقة العربية من البطالة والتي قد تصل الى •r\% عام • • ب، كما ركزت الوثيقة على هجرة خريجي الجامعات العربية الى اوربا وامريكا وغيرها من السلبيات الموجودة في المنطقة العربية، لذا لجأت الولايات المتحدة الى توظيف هذه السلبيات من اجل تدخلها على كافة المستويات السياسية الاقتصادية والثقافية والعسكرية، وصياغة شرق اوسط امريكي وبمساعدة الدول الاوربية، وركزت على تلى

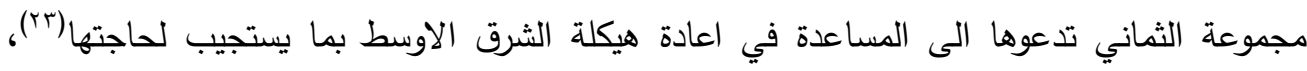
ويبدو ان الولايات المتحدة الامريكية تريد جعل الحكومات العربية مرتبطة بها من خلال تثجيع الديمقراطية في الثرق الاوسط الجديد، كما ان الوعود الامريكية بدعم الحرية في المنطقة العربية وسائر بلدان الثرق الاوسط يشبه الى حد ما وعود دول الوفاق للعرب بتحقيق وحدتهم في اقامة دولة عربية موحدة مقابل الثورة العربية عام 1917 19. 
المؤتمر العلمي الاولي الحادي عشر

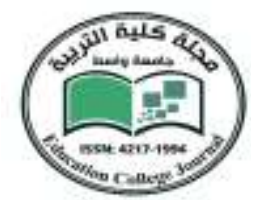

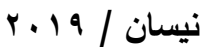

\section{جامعة واسط}

مجلـــة كليــة التربيـة فيسة

ان الثرق الاوسط الجديد الذي تريده الولايات المتحدة الامريكية يجب ان تتنهي فيه الدولة المركزية لتحل محلها الدولة الفيدرالية، بحيث تكون عبارة عن مجموعة من الطوائف او الدذاهب او الاثثيات العرقية، وبذلك تختفي الهوية الوطنية والقومية وتلغى الامة بوصفها انتماء للجماعة البشرية

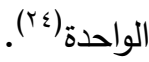

لقد منعت الولايات المتحدة العرب من امتلاك التكنولوجيا الحديثة، ومنعتهح من انشاء اي محطة نووية للاغراض السلمية، وشمل المنع حتى الدول الحليفة لها سواء السعودية او مصر ، وسعت في الوقت نفسه الى انثاء بنك تتمية الثرق الاوسط الجديد، بحيث يكون تمويله من مجموعة الثماني ومن منطقة الثرق الاوسط الجديد، ويبدو ان الهدف من خلق شراكة مالية شرق اوسطية - امريكية لتعزيز العولمة المالية للولايات المتحدة الامريكية(ro). ومن الجدير ذكره بعد الاحتلال الامريكي للعراق دخلت اسرائيل بشكل مكثف، بحيث انشأت اكثر من محطة امنية للموساد الاسرائيلي، فكانت واحدة في بغداد وثانية في كركوك واخرى على الحدود العراقية السورية، فضلا عن تهويد الاقتصاد العراقي وظهور العديد من الثركات الاسرائيلية في

كردستان (Tr)

الخاتمة

تركزت انظار الولايات المتحدة على الثرق الاوسط بصورة عامة والمنطقة العربية بصورة خاصة، ويدو انها اهتمت بالطاقة النفطية بما يتلاءم مع حاجتها الصناعية المتزايدة، فضلا عن منعها الاتحاد السوفييتي من احداث اي اختراق في الخليج العربي، كما انها روجت لثقافة الاحباط والعجز العربي وصولا الى الاستسلام لقرية الهزيمة عن طريق وسائل الاعلام، كذلك دعمها الاصوليات الدينية الهناهضة للحداثة، كما انها سعت الى افتعال الازمات في الدنطقة: الحرب العراقية - الايرانية

$$
\text { (19191-191) (1919 و الازمة العراقية - الكويتية (199). }
$$

تشكل المرحلة الراهنة منعطفا خطيرا بالنسبة للوطن العربي برمته، فالولايات المتحدة الامريكية تحاول الحصول على اسناد دولي واقليمي لمشروعها الاحتلالي للعراق وان نجاحها سيكون نقطة بنة لانطلاق نحو السيطرة على المنطقة العربية برمتها. 
المؤتمر العلمي الاولي الحادي عثر

ن بيسان / 19

جامعة واسط مجلـــة كليـــة التربيـــة

تحاول الولايات المتحدة تبرير شرعية الوجود الاسرائيلي في المنطقة من خلال رفض مفهوم القومية العربية وجعل المنطقة خليط من قوميات وشعوب ولغات، حتى تكون مسألة قيام وحدة فيها بعيدة المنال، مما يجعل لكل قومية لغوية او دينية او عرقية دولتها الخاصة بها، وفي ذلك تكتسب اسرائيل مبرر وجودها وشرعيتها بوصفها قومية لها خصوصيتها في المنطقة وينبغي ان تكون لها دولتها الخاصة بها. الهوامش:

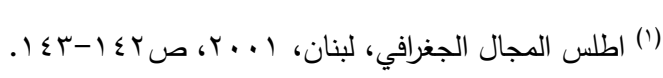

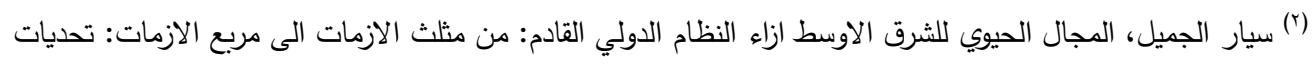

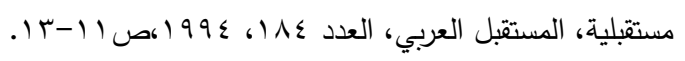

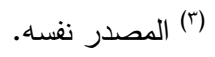

(4) S. Nearing and J. Freeman, Dollar Diplomacy, New York, 1925, P.273.

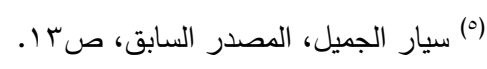

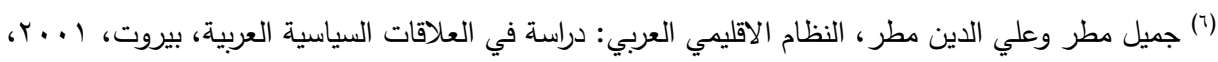
ص (1)

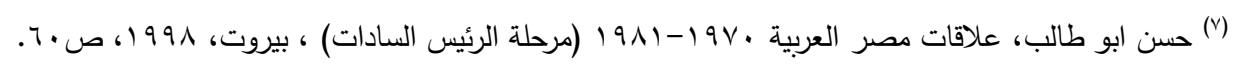

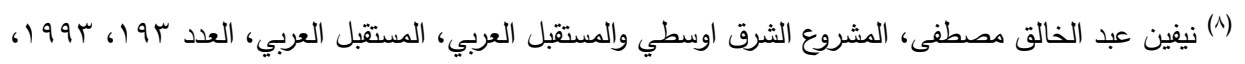
ص V.

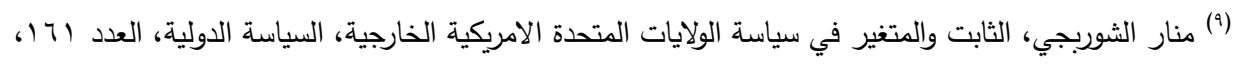

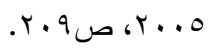

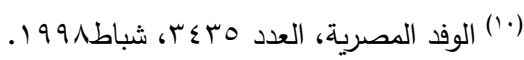

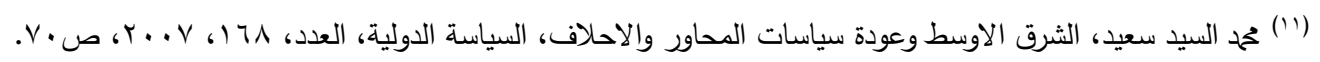
(12) Paul A. Gigot, A great American screw-up: The U.S. and Iraq 1980-1990, National interest, 1990, P.4.

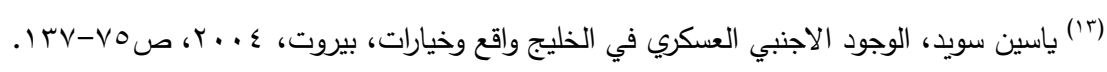

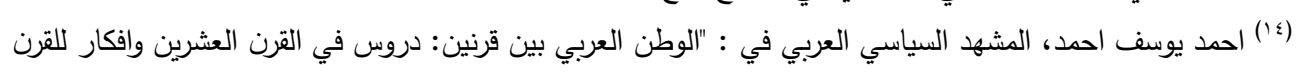

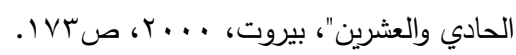

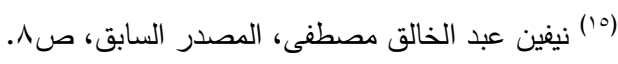

$$
\begin{aligned}
& \text { (17) المصدر نفسه. }
\end{aligned}
$$




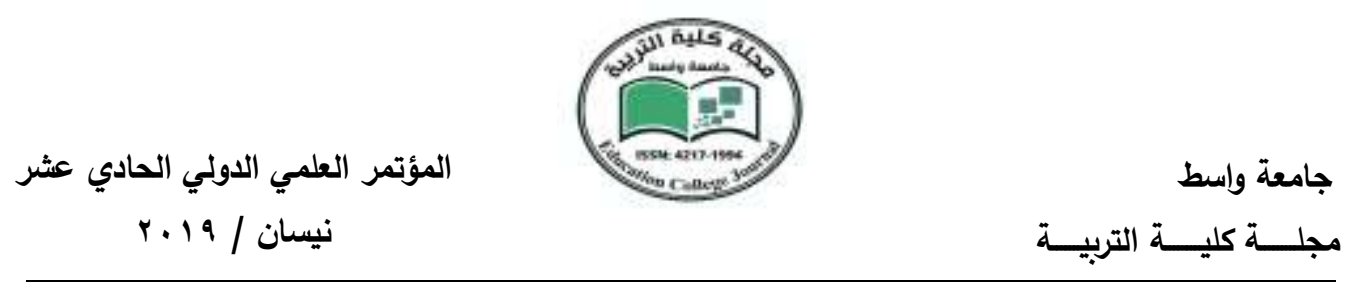

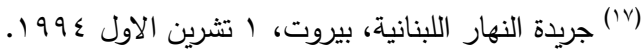

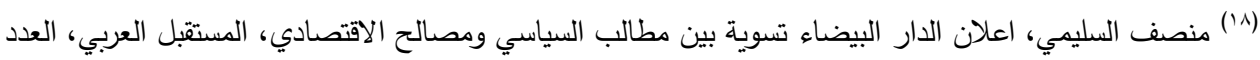

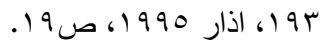

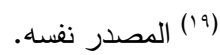

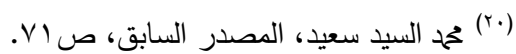
(r) (r)

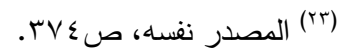

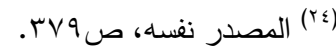

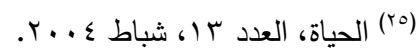

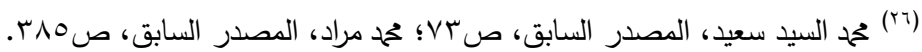

